\title{
Ultra-high energy cosmic rays detected by Auger and AGASA ${ }^{\star}$ Corrections for galactic magnetic field deflections, source populations,
and arguments for multiple components
}

\begin{abstract}
N. M. Nagar and J. Matulich
Astronomy Department, Universidad de Concepción, Concepción, Chile

e-mail: nagar@astro-udec.cl, jmatulich@udec.cl

Received 16 June 2009 / Accepted 24 December 2009

ABSTRACT

Context. The origin and composition of ultra-high energy cosmic rays (UHECRs) remain unclear. Possible sources include active galactic nuclei - selected by various criteria - and extragalactic magnetars.

Aims. We aim to improve constraints on the source population(s) and compositions of UHECRs by accounting for UHECR deflections within existing Galactic magnetic field models (GMFs).

Methods. We used Monte Carlo simulations for UHECRs detected by the Pierre Auger Observatory and AGASA, to determine the UHECR trajectories within the Galaxy and their outside-the-Galaxy arrival directions. The simulations, which used UHECR compositions from protons to iron and seven models of the ordered GMF, accounted for uncertainties in the GMF and a turbulent magnetic field component. Trajectories and outside-the-Galaxy arrival directions were compared with Galactic and extragalactic sources.

Results. For a given proton or light UHECR, the multiple potential outside-the-Galaxy arrival directions within a given GMF model are not very different, allowing meaningful constraints on source populations. Our previous claim of a correlation between a subset of UHECRs and nearby extended radiogalaxies remains valid, even strengthened, within several GMF models. Both the nearest radiogalaxy Cen A, and the nearest radio-extended BL Lac, CGCG 413-019, are potential sources of multiple UHECRs. The correlation appears to be linked to the extended radio source rather than a tracer of an underlying matter distribution. Several UHECRs have trajectories that pass close to the Galactic plane, some passing close to Galactic magnetars and/or microquasars. For heavier UHECRs, the multiple potential outside-the-Galaxy arrival directions of any given UHECR are highly scattered but still allow meaningful constraints. It is possible, but unlikely, that all UHECRs originate in the nearby radiogalaxy Cen A.

Conclusions. Nearby radiogalaxies remain a strong potential source of a significant subset of UHECRs. For light UHECRs, about a third of UHECRs can be "matched" to nearby galaxies with extended radio jets. The remaining UHECRs could also be explained as originating in extended radiogalaxies if one has at least one of: a large UHECR mean free path, a high cluster and/or intergalactic magnetic field, and a heavy composition for two-thirds of the detected UHECRs. If extended radiogalaxies are, or trace, UHECR sources, the most consistent models for the ordered GMF are the BS-S and BS-A models; the GMF models of Sun and collaborators are acceptable if a dipole component is added.
\end{abstract}

Key words. cosmic rays - ISM: magnetic fields - galaxies: active - galaxies: jets

\section{Introduction}

Ultra-high energy cosmic rays (UHECRs) are protons or fully ionized nuclei with energies greater than about $10^{19} \mathrm{eV}$ $(10 \mathrm{EeV})$. On entering the Earth's atmosphere, they produce a shower of secondary particles and excite atmospheric molecules. The detection of both effects from the ground allows a precise measurement of both the initial energy and the arrival direction of the UHECR above the Earth's atmosphere, e.g., by AGASA (Takeda et al. 1999) and the Pierre Auger Observatory (Pierre Auger Collaboration et al. 2004). The resultant positional accuracy of the Earth-arrival direction, now more accurate than a degree, allows correlations with astronomical sources to determine the source population(s) of UHECRs - a long held mystery. Exploiting the full potential of the Earth-arrival directions nevertheless requires taking into account UHECR deflections in Galactic and extragalactic magnetic fields.

Suspected sources of UHECRs include powerful active galaxies, magnetars, core collapse supernovae (SN II), and gamma-ray bursts (for reviews and lectures see Hillas 1999;

* Figures 2, 4 and 5 are only available in electronic form at http://www . aanda.org
Kachelriess 2008 and references therein). That they originate in nearby radiogalaxies and BL Lacs has long been predicted (Rachen \& Biermann 1993; Romero et al. 1996). More exotic explanations include dark matter anhilation and the evaporation of micro black holes. The main energy loss for a UHECR propagating over cosmological distances is expected to be pion-production triggered by interaction with a $\mathrm{CMB}$ photon, the so-called Greisen Zatsepin Kuzmin (GZK) effect (Greisen 1966; Zatsepin \& Kuzmin 1966). The predicted energy loss (around 30\%) and mean free path of the UHECR ( 20$100 \mathrm{Mpc}$ ) depend on the composition and energy of the UHECR and details of the collision process, e.g., the photo-proton cross section (see e.g., Stanev 2004; Kachelriess 2008; PA08).

The Pierre Auger Observatory (PAO) detected 81 UHECR events between 2004 January 1 and 2007 August 31 with reconstructed energies above $40 \mathrm{EeV}$ and zenith angles smaller than $60^{\circ}$. Among these events, 27 have energies higher than $56 \mathrm{EeV}$ and had been reported by the Pierre Auger Collaboration et al. (2007, 2008, hereafter PA07, PA08). The origins of the latter 27 UHECRs, together with 11 UHECRs detected by AGASA at energies above $56 \mathrm{EeV}$ (Hayashida et al. 2000) are the focus of this paper. The PAO has since detected additional UHECRs 
(The Pierre Auger Collaboration et al. 2009a; The Pierre Auger Collaboration et al. 2009b, hereafter PA09a, PA09b) but detailed positions and energies have not yet been published. In this work, we use "UHECR" to refer to UHECRs of energies above $56 \mathrm{EeV}$ unless explicitly mentioned otherwise. The compositions of the detected UHECRs are still disputed (e.g., PA09a; PA09b; Matthews 2007; Hooper \& Taylor 2010) with evidence for a mix of protons and heavy nuclei at the highest energies. There has been some doubt about the measured energies of the UHECRs detected by PAO and AGASA. The PAO team have lowered their previously reported energies by a small percentage (PA09a, PA09b) and there have been suggestions that energies reported by AGASA should be lowered by up to $30 \%$. To avoid confusion, we report our results separately for PAO and AGASA UHECRs. Several other UHECR observatories, e.g., Haverah Park and Fly's Eye, have detected a significant number of UHECRs. Given that the GMF deflection calculations and source matching in this work require accurate energies $(\lesssim 10 \%)$ and arrival directions $\left(\lesssim 2^{\circ}\right)$, we do not include their results in our analysis.

The arrival directions of the 27 UHECRs detected by PAO are not isotropic at a 99\% significance level (PA07, PA08, PA09a, PA09b). Several correlations, or lack thereof, with extragalactic sources have been suggested: AGNs from the catalog of Véron-Cetty \& Véron (PA07, PA08), hard X-ray selected AGNs (George et al. 2008), nearby extended radiogalaxies (Nagar \& Matulich 2008), nearby spiral galaxies (Ghisellini et al. 2008), local volume galaxies (Cuesta \& Prada 2009), and large-scale structure (Ryu et al. 2009).

Galactic and extragalactic magnetic fields deflect cosmic rays via the Lorentz force even at energies above $56 \mathrm{EeV}$. If the extragalactic field is unordered over large scales, the expected deflections at these energies are only a few degrees (e.g., Harari et al. 2002; Dolag et al. 2004). An ordered Galactic magnetic field (GMF) can, however, produce significant deflections (e.g., Takami \& Sato 2008) especially on trajectories that pass close to the Galactic plane and/or for heavy composition UHECRs.

The line-of-sight integrated GMF of the Galaxy has been measured in various directions via e.g., the rotation measures of pulsars and extragalactic radio sources, and the polarization of starlight or radio synchrotron emission (Heiles 1996; Brown et al. 2007; Noutsos et al. 2008; Sun et al. 2008; Han 2009; Beck 2009). In addition, local magnetic fields can be measured accurately by means of the Zeeman splitting of emission lines. For a given line of sight, the rotation measure traces the integrated parallel component of the GMF, while a UHECR is deflected by the perpendicular component of the GMF. A global model of the ordered GMF is thus required to transfer information from rotation measures into information useful for UHECR deflections. Several models of ordered magnetic fields in the Galaxy have been presented. They typically consider one or more of the following: a toroidal type field in and near the Galaxy disk, a toroidal field in the halo, and a poloidal field that reproduces the vertical component seen in the Solar neighbourhood and the Galactic center. Models of the ordered component of the magnetic field are discussed in the next section, and comprehensive reviews can be found in Han (2008) and Beck (2008). Studies of the global magnetic field of other galaxies have greatly facilitated the refinement of models of the Galactic GMF (see Beck 2008 , 2009, for a review), even though there is significant variation between galaxies in both the ordered and turbulent GMF, especially among those with high star formation rates.

In this article, we expand the work of Nagar \& Matulich (2008) in two directions: first we use Monte Carlo simulations to derive UHECR trajectories within the Galaxy and corresponding outside-the-Galaxy arrival directions for all PAO and AGASA UHECRs. The simulations, performed for six UHECR compositions - from protons to iron - and seven GMF models, include uncertainties in the GMF and a turbulent magnetic field component. We then compare the UHECR trajectories within the Galaxy and their outside-the-Galaxy arrival directions with various Galactic and extragalactic sources, and discuss the implications of the results. The individual steps followed in this work have been addressed previously by various authors referenced here and in the next section. The new facet of this work is that we combine all of the above steps within the same Monte Carlo simulation, to model the trajectories of the PAO and AGASA UHECRs with energies greater than $56 \mathrm{EeV}$ within a large number of ordered GMF models and UHECR compositions. Section 2 introduces the magnetic field models and describes the Monte Carlo simulations, Sect. 3 summarizes the sources of the data used, and Sect. 4 describes the principle results obtained. Finally, Sect. 5 presents a brief discussion and the conclusions of our study. Distances to galaxies are calculated using a Hubble constant of $72 \mathrm{~km} \mathrm{~s}^{-1} \mathrm{Mpc}^{-1}$, except for relatively nearby galaxies for which we use the distances that we reference.

\section{Magnetic fields and Monte Carlo simulations}

We consider seven ordered magnetic field models for our Galaxy (for reviews of Galactic and extragalactic ordered magnetic fields see Beck 2008; Han 2008; Beck 2009). Four of these models, the so-called BS-S, BS-A, AS-S, and AS-A have been used by several authors. Here, the first two alphabets signify a (bi)symmetric (BS) or asymmetric (AS) configuration with respect to a transformation between $\theta$ and $\theta+\pi$, where $\theta$ is the cylindrical azimuthal angle in the Galactic plane. The last alphabet signifies whether the field reverses direction (A) or not (B) in passing from above the Galactic plane to below the Galactic plane. Given the measurement of a non-zero vertical $(z)$ component of the GMF, in both the Galactic center and the Solar vicinity, a dipole field model is usually added to the toroidal field above. Detailed descriptions and discussions of these GMFs can be found in Stanev (1997), Beck (2001), Han (2002), Tinyakov \& Tkachev (2002), Prouza \& Šmída (2003), Brown et al. (2007), Kachelrieß et al. (2007), Men et al. (2008), Takami \& Sato (2008), and Han (2009). For these four models, we use the equations and their related normalizations following Takami \& Sato (2008), and always include the dipole field component as specified in Takami $\&$ Sato (2008).

Sun et al. (2008, hereafter S08) presented three new variations of the above GMF models - AS-S+RING, AS-S+ARM, and a modified BS-S - tailored to reproduce observations of Galactic synchrotron emission and its polarization, and the rotation measures of pulsars. For brevity, we sometimes refer to these three models as ASS $+\mathrm{R}, \mathrm{ASS}+\mathrm{A}$, and $\mathrm{BSS}(\mathrm{S})$ in the text and tables. Sun et al. (2008) find that the first two models provide the closest description of current radio observations. These two models use the basic configuration of the AS-S model, but introduce field reversals in a specified ring or arm, respectively. The modified BS-S model is based on the traditional BS-S model but with different parameters and normalizations. The three new models presented by S08 also include a toroidal halo field as presented initially in Prouza \& Šmída (2003), but do not include a dipole magnetic field component. We use these three models and their related halo field described in S08 with only one difference: in the AS-S+ARM model, we directly use the log spiral arm morphology described in Wainscoat et al. (1992) without 
correcting for their shape close to the solar neighbourhood as in S08, who used the correction suggested in Taylor \& Cordes (1993). We do not expect this small change in the morphology of the spiral arms to cause significant changes in the UHECR trajectories. Sun et al. (2008) assumed that there is no ordered dipole field component in the Galaxy GMF, arguing that the vertical $(z)$ component of the local magnetic field is a turbulent component. Since the parametric form and normalizations of the S08 models were derived by fitting radio observations, it is not strictly correct to add a dipole field to the models. Nevertheless, for illustrative purposes (and out of curiosity) we also performed simulations of the S08 GMF models including the dipole field component as specified in Takami \& Sato (2008). We refer to these results as those of the S08 GMFs plus dipole.

We simulated UHECR trajectories for six different UHECR compositions: protons, $\mathrm{He}^{2+}, \mathrm{O}^{8+}, \mathrm{Al}^{13+}, \mathrm{Ca}^{20+}$, and $\mathrm{Fe}^{26+}$. Once chosen, a composition was kept fixed throughout the full trajectory within the Galaxy: i.e., we do not consider photodissociation-produced composition changes to a UHECR during its Galactic trajectory. UHECR trajectories were simulated by firing an antiparticle with the corresponding UHECR mass, (anti)charge, and energy from the Earth in the Earth-arrival direction of the UHECR and following its trajectory - including deflections by the magnetic field - in steps of $10 \mathrm{pc}$. The simulations were carried out until the antiparticle left a sphere of diameter $40 \mathrm{kpc}$ centered on the Galactic center. The Galactic coordinate pair $(l, b)$ derived from the final position and velocity of the antiparticle in the simulation is equivalent to the arrival direction of the UHECR before it entered the Galaxy, and we refer to this direction as the "outside-the-Galaxy" arrival direction, to distinguish it from the 'Earth-arrival' direction measured by the observatory.

Each UHECR's trajectory within each GMF model was simulated fifty times using a Monte Carlo approach in which we introduced both uncertainties in the ordered magnetic field and a random turbulent magnetic field component. In more detail, in each $10 \mathrm{pc}$ simulation step, we first computed the 3 -vector representing the cartesian coordinates of the ordered magnetic field from the relevant model. For the AS-S, AS-A, BS-S, and BS-A models this was the sum of the cylindrical (disk) field and the dipole field. For the AS-S+ARM, AS-S+RING, and BS-S model of S08 this was the sum of the disk and halo fields (with or without the dipole field as described above). We then added a Gaussian-distributed random error in each component of the ordered magnetic field, i.e., a change in both magnitude and direction of the ordered field. This was performed by adding to the 3 -vector of the previous step, a 3-vector whose every component was a random number derived from a Gaussian distribution of mean $=0$ and standard deviation $\sigma=0.5$ times the value of the corresponding cartesian component of the GMF. Finally, we added a turbulent field component. The turbulent field component was computed following the recipe described in Prouza \& Šmída (2003) with slightly different normalization parameters: the maximum turbulent magnetic field strength in each cartesian component was taken to be $\pm 10 \mu \mathrm{G}$, and the probability of a turbulent field being present was taken to be $1 \%$ in the halo of the Galaxy, 20\% in the disk of the Galaxy (Galactic radius less then $20 \mathrm{kpc}$ and Galactic height less than $1.5 \mathrm{kpc}$ ) and $80 \%$ in the spiral arms of the Galaxy - where we used the spiral arm definition from Wainscoat et al. (1992), spiral arm thickness $0.75 \mathrm{kpc}$ in the disk (i.e., coordinate $r$ ), and half-height $0.5 \mathrm{kpc}$ (coordinate $z$ ). We therefore generated a random number to determine whether a turbulent field should be added following the probabilities above. If so, we generated a 3 -vector in which each component was a random number uniformly distributed between $-10 \mu \mathrm{G}$ and $10 \mu \mathrm{G}$. This was added to the magnetic field 3 -vector in the previous step to derive the total magnetic field used in the deflection calculation of this step. This turbulent field was then kept constant for the next 50 pc (i.e., an additional five simulation steps), which is the expected scale length of the turbulent field in the Galaxy (Prouza \& Šmída 2003; Beck 2008). We note that the turbulent field used here is relatively large compared to current measurements of the turbulent field in the Galaxy (S08) and other galaxies (Beck 2009).

To summarize, within each GMF model we derive 50 outside-the-Galaxy arrival directions for each UHECR of a specific composition, or a total of 300 outside-the-Galaxy arrival directions for each UHECR over all six compositions modelled.

\section{Data}

Positions and energies of the 27 UHECRs detected by the PAO with energies $\geq 56 \mathrm{EeV}$ were taken from PA08; we did not make the small energy corrections to these UHECRs later reported by the PAO (PAO09b). Positions and energies of the UHECRs detected by AGASA with energies $\geq 56 \mathrm{EeV}$ were taken from Hayashida et al. (2000), which is an updation of the data listed in Takeda et al. (1999). For AGASA UHECRs, we lowered the energies reported in Takeda et al. (1999) by 30\%, resulting in 11 UHECRs with energy above $56 \mathrm{EeV}$. In this work, we use "AGASA UHECRs" to refer to these 11 UHECRs only unless explicitly stated otherwise.

Our catalogs of astronomical sources were drawn from various publications and web-based source lists, and chosen to be representative of previously suspected or posited Galactic and extragalactic sources of UHECRs. In all cases, we attempted to select the most comprehensive catalog available. The source lists of galaxies, galaxy clusters, GRBs, Galactic supernova remnants, and extragalactic radio supernova are described in Nagar \& Matulich (2008). As in Nagar \& Matulich (2008), in the case of radiogalaxies and radio sources we used several surveys and catalogs, including NVSS (Condon et al. 1998), SUMMS (Bock et al. 1999), and NED. The list of extragalactic jets is based on that compiled by Liu \& Zhang (2002), to which we added data from NED and remeasured the total flux and total extent of the radio emission from either the NVSS and SUMMS maps or the list of DRAGNs compiled by Leahy ${ }^{1}$. The DRAGNs list represents the subset of the 3CRR sources (Laing et al. 1983) that have one of or all of radio-emitting jets, lobes, and hotspots. The total extent of the radio emission therefore includes both radio jets and any radio lobes. This extent is typically referred to as the largest angular size (LAS) or largest linear size (LLS). In the case of double-sided jet or lobe sources, we added the LLS of the two jets and lobes as scalars instead of vectors to discount the effects of jet and lobe bending. Nagar \& Matulich (2008) listed the 10 radiogalaxies in the field of view of PAO with $D \leq 75 \mathrm{Mpc}$ and LLS $\geq 180 \mathrm{kpc}$. To this, we added all other (northern) radiogalaxies satisfying the same criteria: NGC 315 $(D=69.5 \mathrm{Mpc} ; L L S=1111 \mathrm{kpc}), \operatorname{NGC} 383(D=71.7 \mathrm{Mpc}$; $L L S=907 \mathrm{kpc}), \operatorname{NGC} 1275(D=74.1 \mathrm{Mpc} ; L L S=468 \mathrm{kpc})$, NGC $5127(D=68.3 \mathrm{Mpc} ; L L S=230 \mathrm{kpc})$, and CGCG 514$050(D=72 \mathrm{Mpc} ; L L S=506 \mathrm{kpc})$. Together, these 15 galaxies form our sample of all nearby $(D \leq 75 \mathrm{Mpc})$, extended $(L L S \geq 180 \mathrm{kpc})$ radiogalaxies. For nearby galaxies, we also used the HIPASS catalog (Meyer et al. 2004; Wong et al. 2006) used by Ghisellini et al. (2008) in their study of the correlation

${ }^{1}$ http://www.jb.man.ac.uk/atlas/dragns.html 


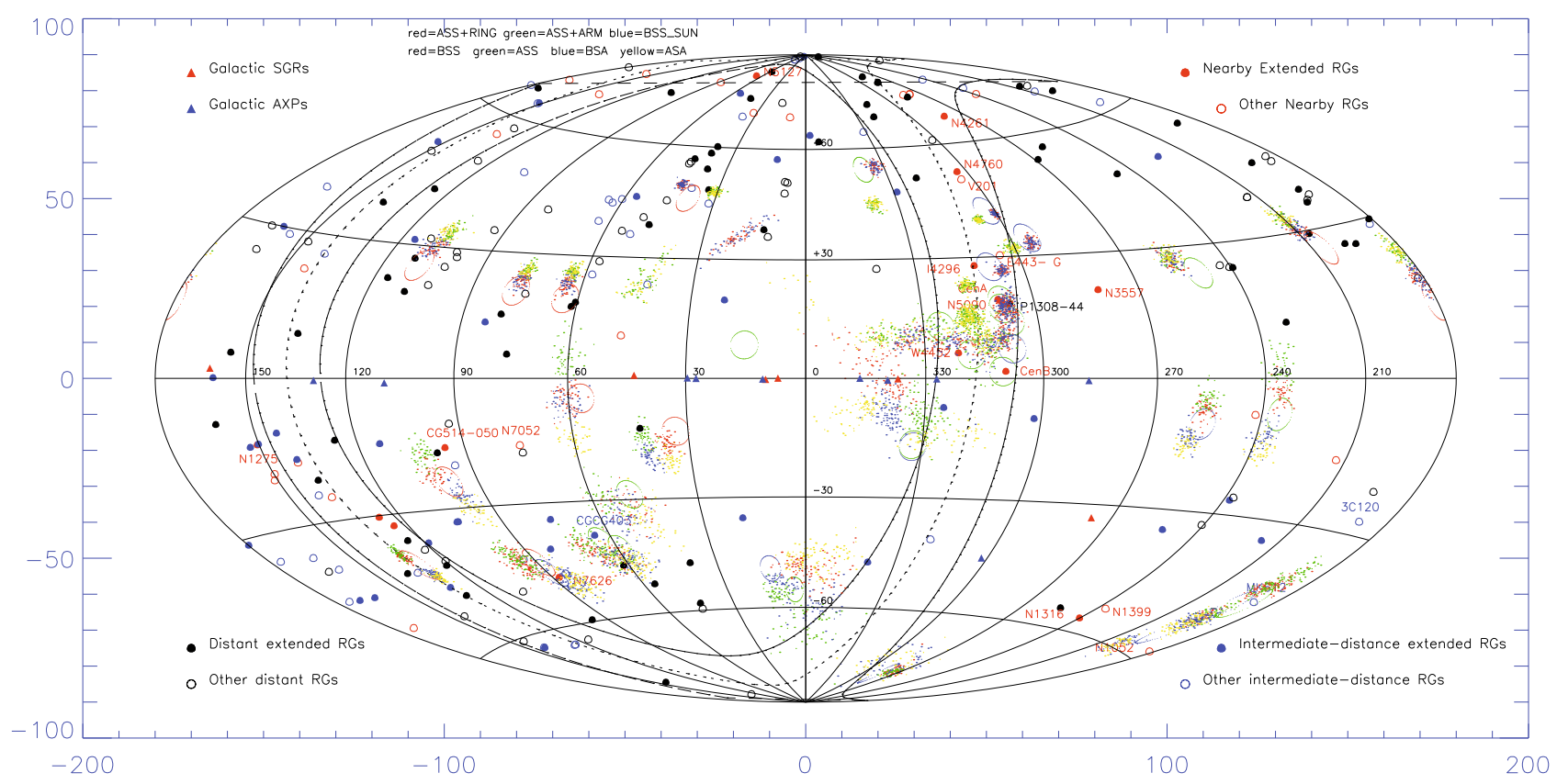

Fig. 1. A comparison of the Earth-arrival directions of all UHECRs with energies above $56 \mathrm{EeV}$ detected by PAO (blue for UHECRs with energies $\geq 75 \mathrm{EeV}$ and green for UHECRs with energies between 56 and $75 \mathrm{EeV}$ ) and AGASA (red open circles of radius $3.5^{\circ}$ ) with the estimated outsidethe-Galaxy arrival directions of the same UHECRs (small colored dots) for our Monte Carlo simulations of the BS-S (red), AS-S (green), BS-A (blue), and AS-A (yellow) GMFs using a proton composition for the UHECRs. The colored circular symbols indicate the positions of galaxies with radio jets at $D \leq 75 \mathrm{Mpc}$ (red), $75 \mathrm{Mpc}<D \leq 200 \mathrm{Mpc}$ (blue), and $200 \mathrm{Mpc}<D \leq 500 \mathrm{Mpc}$ (black). In all redshift bins, filled circular symbols are used for galaxies with radio structures more extended than $180 \mathrm{kpc}$, and open circular symbols for galaxies with radio structures less extended than $180 \mathrm{kpc}$. Galactic SGRs (red triangles) and AXPs (blue triangles) are also plotted. The Supergalactic plane is marked by the dashed line. In this and following figures we use an Aitoff-Hammer (equi-area) projection in Galactic coordinates.

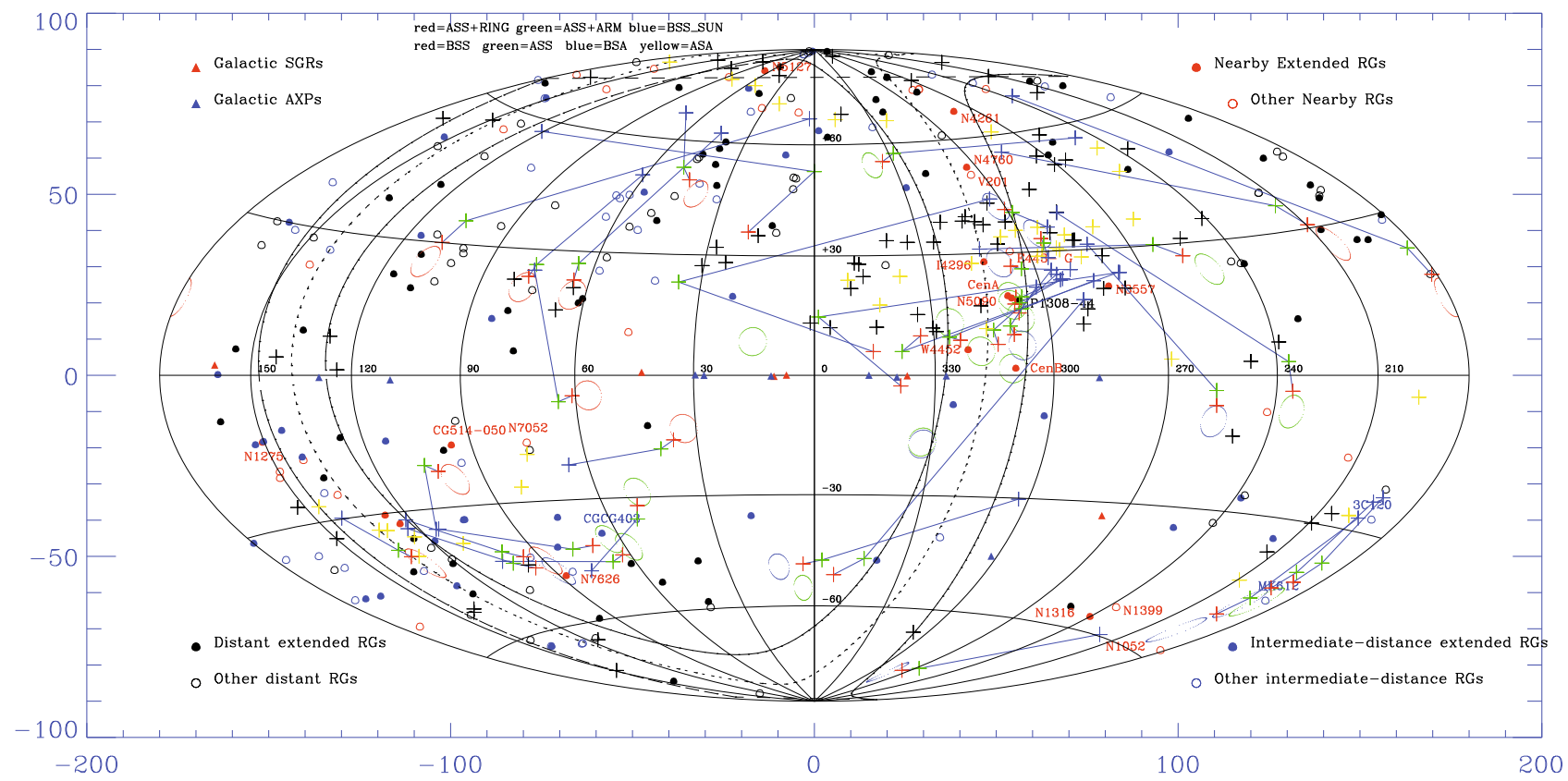

Fig. 3. A comparison of the measured Earth-arrival directions of all UHECRs with energies above $56 \mathrm{EeV}$ detected by PAO and AGASA (blue, green, and red open circles with radius $3.5^{\circ}$ as in Fig. 1) with the estimated outside-the-Galaxy arrival directions of the same UHECR (crosses) within the BS-S model for UHECR compositions of protons (red), He (green), oxygen (blue), aluminum (yellow), and calcium and iron (black). The first three compositions of the same UHECR are connected with blue lines, while the heavier compositions are not connected to avoid plot overcrowding. Other symbols and names in the figure are the same as in Fig. 1.

between UHECR arrival directions and nearby galaxies. In addition, we used the revised Third Reference Catalog of Bright Galaxies (Corwin et al. 1994) to select a sample of nearby elliptical galaxies.
In this work, we consider several new source catalogs: Galactic soft gamma-ray repeaters (SGRs) and Galactic anomalous X-ray pulsars (AXPs) were taken from Woods \& Thompson (2006) and updated with the online list maintained by the McGill 
pulsar group ${ }^{2}$. As of June 2009, this list includes six SGRs (two of which are candidates) and 10 AXPs (one of which is a candidate). These SGRs and AXPs are believed to be high magnetic field radio pulsars or "magnetars". Of the total of 16 SGRs and AXPs, 14 are close to the plane of the Galaxy, and two are in the SMC and LMC.

Confirmed Galactic microquasars were taken from the list of Paredes (2005), and candidate micro-quasars from Combi et al. (2008). Various gamma-ray catalogs were used: the BeppoSAX catalog of GRB and X-ray afterglows (de Pasquale et al. 2006), the BeppoSAX complete catalogue of GRBs (Vetere et al. 2007), gamma-ray blazars in the northern sky (Sowards-Emmerd et al. 2003), blazar counterparts for 3EG sources (Sowards-Emmerd et al. 2004), gamma-ray blazar candidates (Sowards-Emmerd et al. 2005), sources detected by ISGRI (Bodaghee et al. 2007), and the Third EGRET catalog (Hartman et al. 1999). We also used the HESS (Hofmann 2005) catalog of 54 gamma-ray sources between $100 \mathrm{GeV}$ and $100 \mathrm{TeV}$ as obtained from the HESS online catalog in June $2009^{3}$.

\section{Results}

We performed several Monte Carlo simulations in which we varied the magnitudes of the error of the ordered field and the maximum turbulent field strength. Here we present and discuss the results of simulations in which we used a Gaussian-distributed (mean $=0$ and standard deviation $\sigma=50 \%$ of the value of the cartesian GMF component) error in each cartesian component of the ordered GMF, and a turbulent magnetic field uniformly distributed between $-10 \mu \mathrm{G}$ and $10 \mu \mathrm{G}$ in each cartesian component of the magnetic field, with details as described in Sect. 2. We note that for the simulated GMF error, using $25 \%-100 \%$ instead of $50 \%$ as described above does not significantly change the trajectories of antiprotons. The relative deflections experienced by the UHECR from the ordered field and turbulent field components can thus be roughly judged by comparing the average and rms of the deflection angles corresponding to the fifty outsidethe-Galaxy arrival directions for each UHECR within each GMF model.

We used the following distance limits between a single outside-the-Galaxy arrival direction and an extragalactic astronomical object to be considered a "match": $2.5^{\circ}$ for $\mathrm{H}, 3^{\circ}$ for $\mathrm{He}, 6^{\circ}$ for $\mathrm{O}, 9^{\circ}$ for $\mathrm{Al}, 12^{\circ}$ for $\mathrm{Ca}$, and $15^{\circ}$ for $\mathrm{Fe}$. These semiarbitrary values were chosen by considering a constant additive error of $2^{\circ}$ - to account for e.g., the error in determining the arrival direction of the UHECR - plus a composition-weighted error of 0.5 times the charge of the UHECR, which accounts roughly for errors in the deflection calculations and deflections by intergalactic magnetic fields but avoids too large "match radii" for heavy nuclei. To identify an astronomical source with a UHECR, we require that at least 4 of the 50 outside-the-Galaxy arrival directions are "matched" by the above criterion; this $\geq 8 \%$ match probability was chosen because it roughly implies that a match cannot be ruled out at greater than the $2 \sigma$ level for the simulation parameters.

Graphical representations of our simulation results are shown in Figs. 1 to 5. Coordinates of the arrival directions of the UHECRs detected by PAO (blue for UHECRs with energy $\geq 75 \mathrm{EeV}$ and green for UHECRs with energy between 56 and

\footnotetext{
2 http://www.physics.mcgill.ca/ pulsar/magnetar/main. html

${ }^{3}$ http://www .mpi-hd.mpg.de/hfm/HESS/pages/home/ sources/
}

$75 \mathrm{EeV}$ ) and AGASA (red) are shown as open circles of (arbitrary) radius $3.5^{\circ}$. The outside-the-Galaxy arrival directions of the UHECRs are plotted with small colored dots $(50$ per UHECR, each representing the result of one Monte Carlo simulation). The outside-the-Galaxy arrival directions were plotted in the order red, green, blue, and yellow. When a color is not shown for a UHECR, the data are below that of one of the following colors in the sequence above. For example, the two pairs BS-S (red) and BS-A (blue) and AS-S (green) and AS-A (yellow) have the same deflection for positive values of Galactic latitude $(b)$. To avoid overcrowding, results for the three models of S08 are shown separately from the other four models.

Figures 1 to 5 include the positions of all galaxies with radio jets from the catalog of Liu \& Zhang (2002) within $500 \mathrm{kpc}$ and the 16 confirmed and candidate SGRs and AXPs in the Galaxy (Woods \& Thompson 2006). As in Nagar \& Matulich (2008) but using different cutoffs, we divided the galaxies with radio jets into three redshift bins: "nearby" $(D \leq 75 \mathrm{Mpc}$; red circular symbols), "intermediate" (75 Mpc $<\mathrm{D} \leq 200 \mathrm{Mpc}$; blue circular symbols), and "distant" (200 Mpc $<\mathrm{D} \leq 500 \mathrm{Mpc}$; black circular symbols). In all redshift bins, we distinguish between galaxies with radio structures more extended than $180 \mathrm{kpc}$ ("extended"; solid symbols) and those with radio structures less extended than $180 \mathrm{kpc}$ ("compact"; open symbols). Filled triangles are used for Galactic magnetars: red for SGRs and blue for AXPs. Of the 16 magnetars, two are in the LMC and SMC and the others are close to the plane of the Galaxy.

\subsection{UHECR trajectory simulations: protons}

Columns 5 to 7 of Tables 1 and 2 present the results of the deflection simulations for proton UHECRs within the BS-A GMF model. Tabular results for other models are not shown for space reasons and are available on request from the authors. The first four columns of the tables identify the UHECR by an index number, its arrival direction in Galactic coordinates, and its energy. Then, for each UHECR composition simulated, we list the average and rms of the deflection angles corresponding to the 50 outside-the-Galaxy arrival directions of each UHECR, followed by the nearby extended radiogalaxy that most closely matches the fifty outside-the-Galaxy arrival directions (see the previous section for a definition of a match). A nearby extended radiogalaxy is only listed if more than four of the 50 outside-theGalaxy arrival directions are matched. For proton UHECRs, the average deflection and its rms for each UHECR are both typically $1^{\circ}-5^{\circ}$, though values greater than $30^{\circ}$ are seen for a few UHECRs whose trajectories pass close to the Galactic plane and/or Galactic center.

We emphasize four important results of the proton-UHECR trajectory simulations:

(a) Even after considering errors in the model GMF and adding a relatively strong turbulent magnetic field component, the outside-the-Galaxy arrival directions of the majority of proton-UHECRs are typically sufficiently concentrated to allow meaningful comparisons with Galactic and extragalactic sources;

(b) For a given proton-UHECR, the outside-the-Galaxy arrival directions are typically significantly different for different GMF models. An assumption of the source population therefore allows constraints on competing models of the ordered GMF.

(c) Almost all UHECRs with Galactic latitude $-25^{\circ}<b<0^{\circ}$ have trajectories that pass close to or cross the Galactic plane 


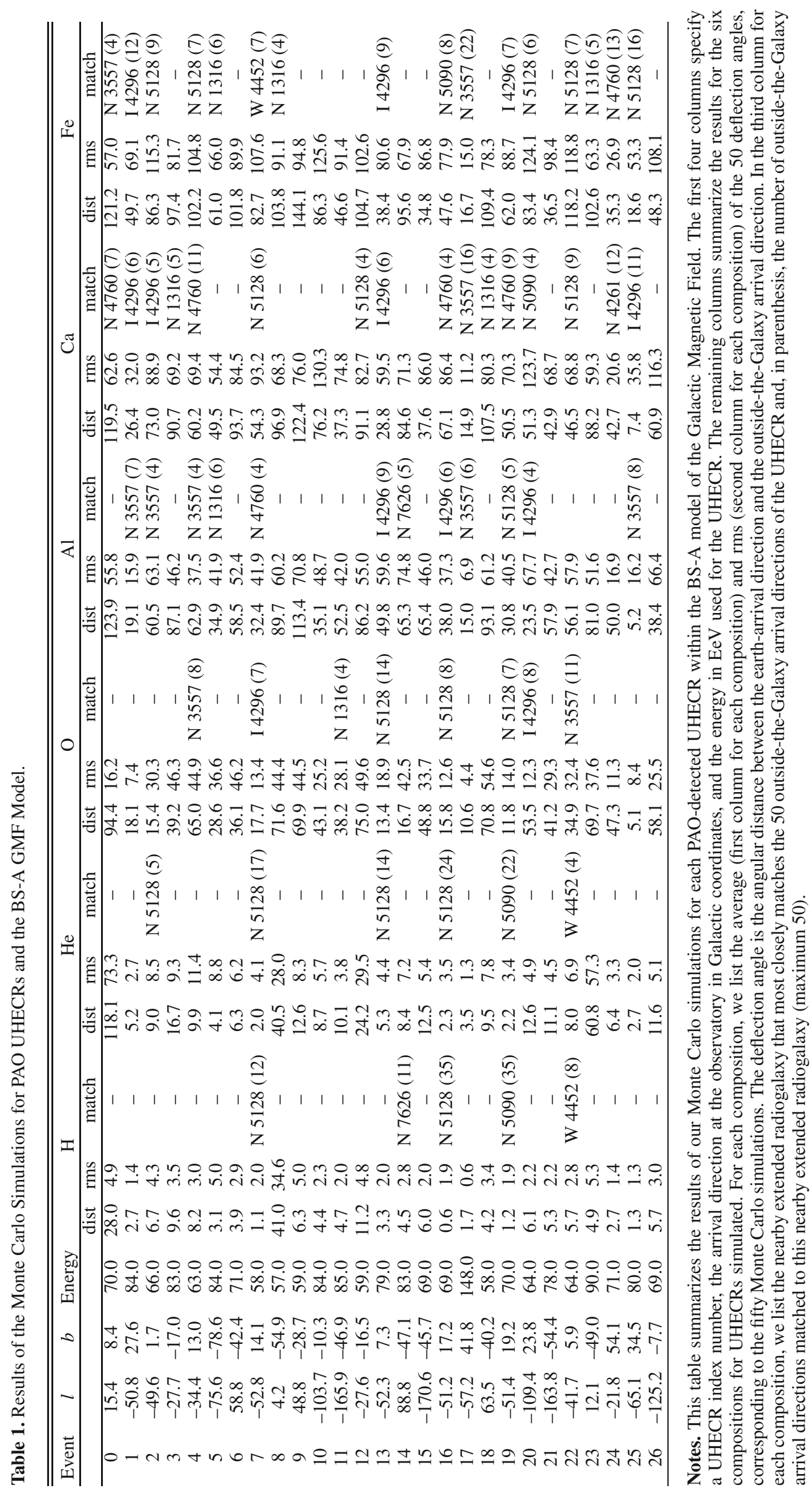




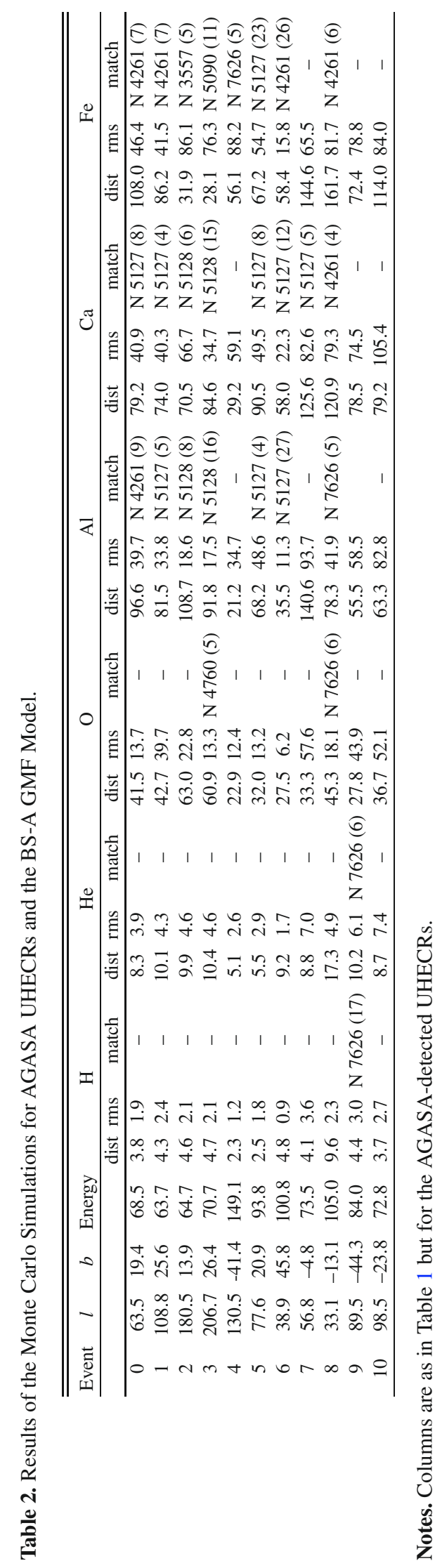

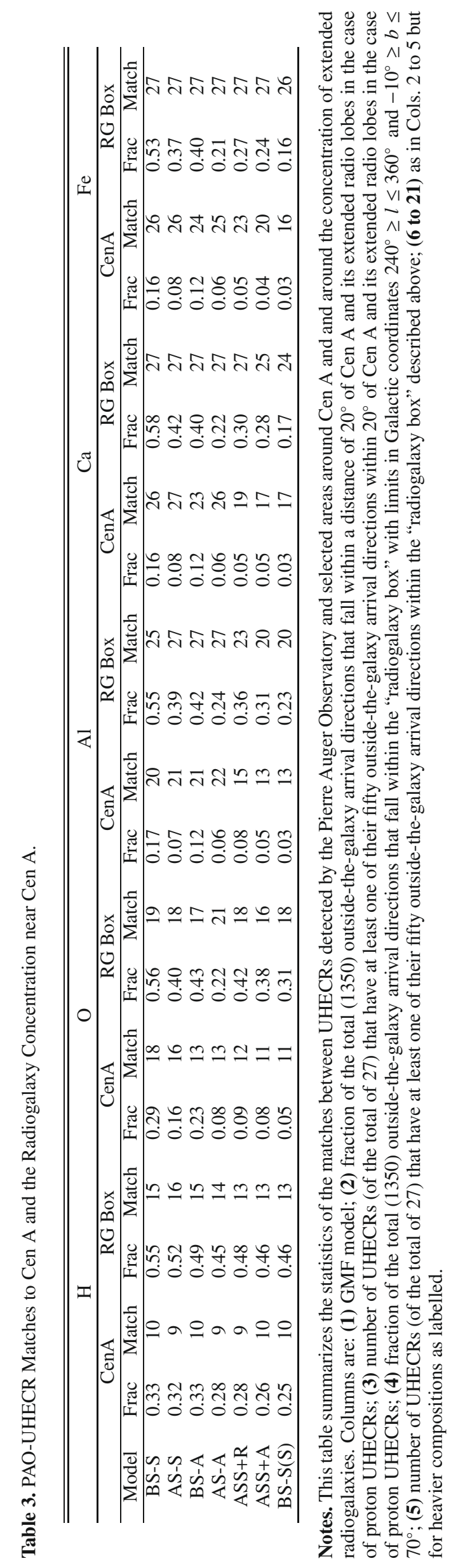

Page 7 of 13 


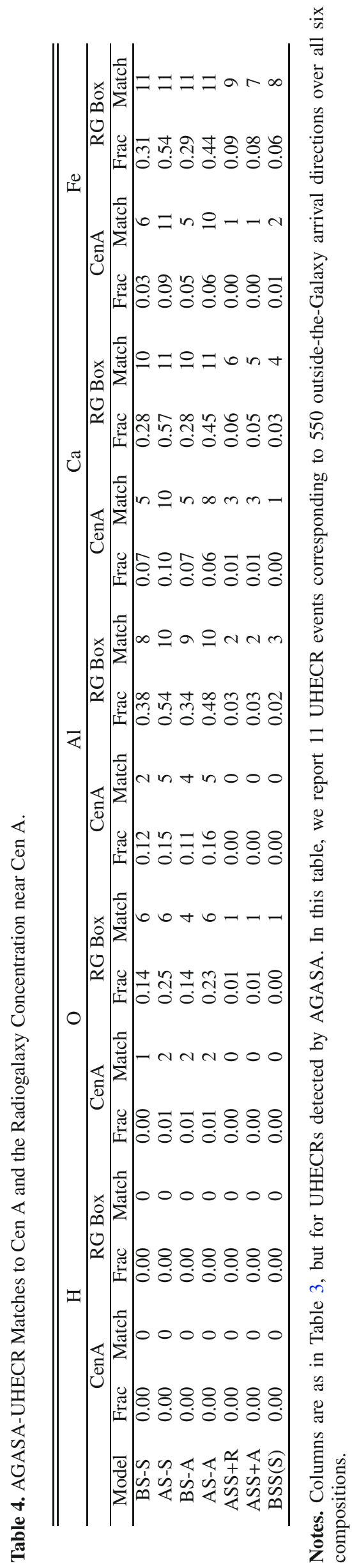

within certain GMF models, especially those which include an ordered dipole field.

(d) As discussed in detail in Sect. 4.5, the match between proton-UHECRs and nearby extended radiogalaxies (Nagar \& Matulich 2008) is maintained in most GMF models, and significantly strengthened in some GMF models.

\subsection{UHECR trajectory simulations: heavy nuclei}

With increasingly heavy UHECR compositions, the increased deflections experienced by the UHECRs tend to scatter their out-of-Galaxy arrival directions over larger regions of the sky. Figure 3, which compares the deflections experienced by UHECRs in four models for different UHECR compositions, emphasizes the importance of UHECR composition for source identification. The simulation in this figure does not include a Monte Carlo approach: i.e., we did not consider GMF uncertainties or turbulent magnetic fields. We point out several interesting features in Fig. 3, especially for oxygen compositions:

(a) the three PAO UHECRs near $l=200^{\circ}, b=-45^{\circ}$ have out-of-Galaxy arrival directions close to the direction of the radiogalaxy 3C 120 for oxygen composition;

(b) the twin mystery PAO UHECRs close to $l=335^{\circ}, b=-18^{\circ}$ have out-of-Galaxy arrival directions close to the Galactic plane for light compositions, and out-of-Galaxy arrival directions towards the Cen $\mathrm{A}$ concentration of radiogalaxies for an oxygen composition; and

(c) the out-of-Galaxy arrival directions of several UHECRs are close to the direction of the extended radiogalaxies NGC 315 and NGC 383 (near position $l=130^{\circ}, b=-30^{\circ}$ ) for an oxygen composition.

A summary of the results of our Monte Carlo simulations for heavy-nuclei UHECRs within the BS-A GMF model can be found in Tables 1 and 2. Graphical representations of oxygen and iron UHECRs within the BS-S, BS-A, AS-S, and AS-A models are presented in Figs. 4 and 5. As expected, both the average and the rms of the deflection angles corresponding to the 50 outsidethe-Galaxy arrival directions for each UHECR increase with increasing UHECR mass (Tables 1 and 2). For compositions up to around oxygen, the 50 outside-the-Galaxy arrival directions corresponding to each UHECR typically still appear to be together on the sky - allowing potential source searches (Fig. 4). For compositions close to and higher than that of calcium, the fifty outside-the-Galaxy arrival directions corresponding to each UHECR are typically scattered over most of the sky. However, even in the case of iron UHECRs (Fig. 5) a concentration can still be seen in the region of the SuperGalactic plane near to and north of Cen A.

\subsection{Source populations: Centaurus A}

The concentration of UHECRs in the Cen A region has attracted much attention since its first report by the PAO team. Our Monte Carlo simulations can be used to test whether Cen A is the dominant, or even only, source of UHECRs. The results of these tests are shown in Table 3 for UHECRs detected by PAO and Table 4 for UHECRs detected by AGASA. In these tables, the first column for each composition reports the fraction of the total (1350 for PAO and 550 for AGASA) outside-the-Galaxy arrival directions that fall within three $20^{\circ}$ circles centered on the Cen A nucleus and its northern and southern radio lobes. The second column for each composition shows the number of UHECRs (of 
N. M. Nagar and J. Matulich: UHECRs: galactic magnetic field deflections
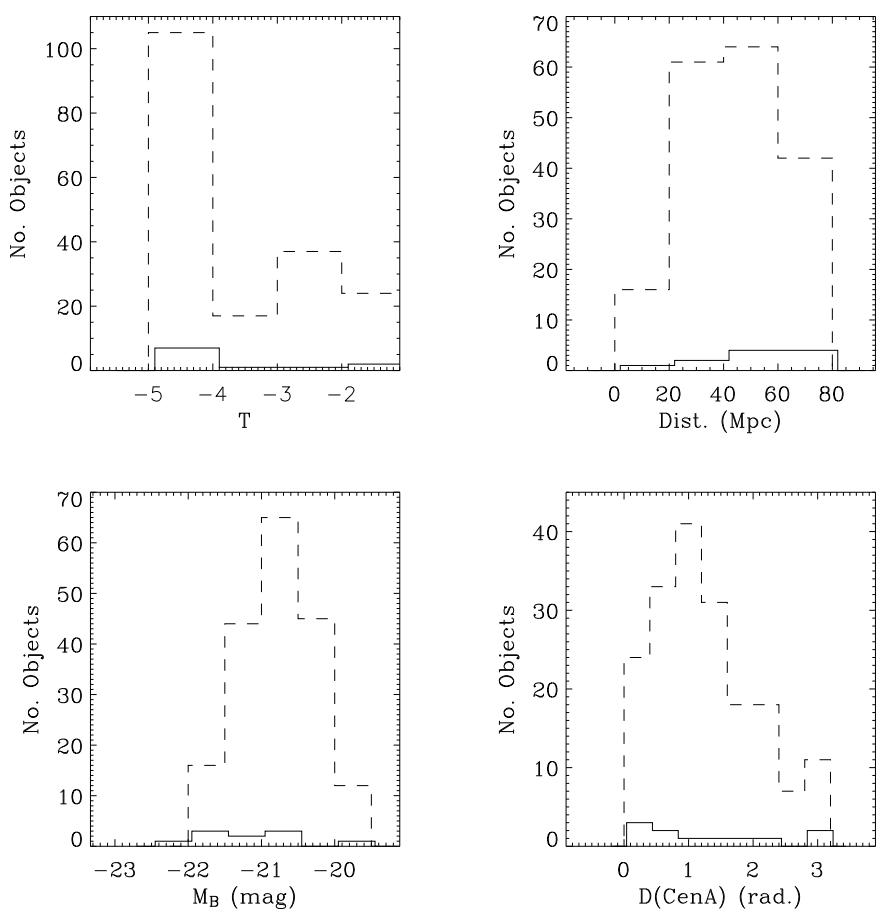

Fig. 6. Histograms of morphological type, distance, absolute $B$ magnitude, and distance from Cen A for the two samples of $D \leq 75 \mathrm{Mpc}$ elliptical galaxies drawn from the RC3 catalog (see text): the 11 nearby RC3 ellipticals with extended radio structures (solid line histograms) and the matched control sample of 183 nearby RC3 ellipticals without extended radio structures.

the total of 27 for PAO and 11 for AGASA), for which at least one of the fifty outside-the-Galaxy arrival directions falls in the $20^{\circ}$ circles described above.

Within our simulation parameters, it is possible but unlikely that Cen A is the source of all PAO-detected UHECRs: for proton UHECRs, one third or less of the total outside-the-Galaxy arrival directions are within the $20^{\circ}$ circles around Cen $\mathrm{A}$ in any GMF model, and almost two thirds of PAO UHECRs do not even have one outside-the-Galaxy arrival direction within the above area. For heavier compositions, a smaller fraction of total outside-the-Galaxy arrival directions are within the $20^{\circ}$ circles around Cen A but a larger fraction of PAO UHECRs, almost $100 \%$ for $\mathrm{Ca}$ and $\mathrm{Fe}$, have at least one outside-the-Galaxy arrival direction that falls within the Cen A circles. The latter result is caused by the large scatter in the outside-the-Galaxy arrival directions of any given heavy composition UHECR. In the case of AGASA UHECRs, the fraction of outside-the-Galaxy arrival directions that fall within the Cen A circles is very small for all compositions, though for heavy UHECR compositions, a large fraction or all UHECRs have at least one outside-theGalaxy arrival direction falling within the Cen A circles.

If we consider all 300 outside-the-Galaxy arrival directions corresponding to each UHECR (6 compositions, 50 outside-theGalaxy arrival directions per composition), then within the models AS-S, AS-A, BS-S, and BS-A, all PAO-detected UHECRs have at least one outside-the-Galaxy arrival direction that falls within the $20^{\circ}$ circles around Cen A. In the S08 GMF models, a few (two to six) PAO UHECRs do not satisfy the above criterion. For the AGASA-detected UHECRs, all UHECRs in the AS-S and AS-A models have at least one outside-the-Galaxy arrival direction that falls within the $20^{\circ}$ circles around Cen A for one composition, while in the other models between two and eight UHECRs do not satisfy this criterion.

\subsection{Source populations: the radiogalaxy concentration around Cen $A$}

As Nagar \& Matulich (2008) pointed out, the area around Cen A hosts the highest density of nearby extended radiogalaxies. We repeated the analysis of the previous section but using a box around all nearby extended radiogalaxies in this area. For simplicity, we used the following limits for the box: Galactic longitude between $240^{\circ}$ and $360^{\circ}$ and Galactic latitude between $-10^{\circ}$ and $70^{\circ}$ (the "RG box"). The results are listed in sub-columns 3 and 4 for each composition in Table 3 for PAO UHECRs and Table 4 for UHECRs detected by AGASA.

The "RG box" contains between a quarter and a half of all outside-the-Galaxy arrival directions for any one given composition. For light UHECRs, at least half of all PAO-detected UHECRs have at least one outside-the-Galaxy arrival direction that falls in the RG box. For $\mathrm{Ca}$ and $\mathrm{Fe}$ compositions, all UHECRs satisfy this criteria. In the case of AGASA UHECRs, heavy compositions are required to provide a good match between the UHECRs and the RG box.

If we consider all 300 outside-the-Galaxy arrival directions corresponding to each UHECR then within the models AS-S, AS-A, BS-S, and BS-A, all PAO and AGASA UHECRs have at least one outside-the-Galaxy arrival direction that falls within the RG box. In the S08 GMF models, a few (two to four) AGASAdetected UHECRs do not satisfy the above criterion.

\subsection{Source populations: nearby extended radiogalaxies}

Nagar \& Matulich (2008) argued that the arrival directions of a subset of PAO-detected UHECRs are correlated with the directions of nearby extended radiogalaxies. A distance of smaller than $3.5^{\circ}$ was found between the radio structures of six extended radiogalaxies (of the 10 "visible" to the PAO) and 8 UHECRs detected by PAO. Considering the full sky, we have a total of 15 nearby $(D<75 \mathrm{Mpc})$ galaxies with extended $(>180 \mathrm{kpc})$ radio structures, the new galaxies being listed in Sect. 3, to be compared to 38 UHECRs. Adding the AGASA-detected events provides two new matches to Earth-arrival directions of UHECRs: an AGASA event with original reported energy $E=120 \mathrm{EeV}$ matched to NGC 7626 (which is also matched to a PAO-detected UHECR) and a match between an AGASA event with original reported energy $E=68 \mathrm{EeV}$ with CGCG 514-050 (this event does not appear in the figures because its $30 \%$ reduced energy is below our UHECR cutoff of $56 \mathrm{EeV}$ ). The correlation between nearby extended radiogalaxies and UHECRs detected by PAO and AGASA therefore remains highly statistically significant even before considering deflections by the GMF. We note that the Supergalactic plane in the Cen A region passes close to the Galactic longitudes where the deflection produced by an ordered GMF with azimuthal symmetry is minimal (e.g., Takami $\&$ Sato 2008). It may be this fortuitous coincidence that allowed us to identify several UHECR events with nearby extended radiogalaxies in Nagar \& Matulich (2008).

Including deflections of UHECRs by the GMF maintains, and in some models strengthens, the correlation between UHECRs and nearby extended radiogalaxies. In its first four columns, Table 5 lists the statistics of matches between UHECRs and our sample of 15 nearby extended radiogalaxies for all compositions and models simulated. The BS-S and BS-A models clearly provide the closest match between nearby extended radiogalaxies and UHECRs. For proton UHECRs, the matches are few, in part because of the use of a rather strict match criterion 
Table 5. Matches between PAO-UHECRs \& Nearby Elliptical Samples.

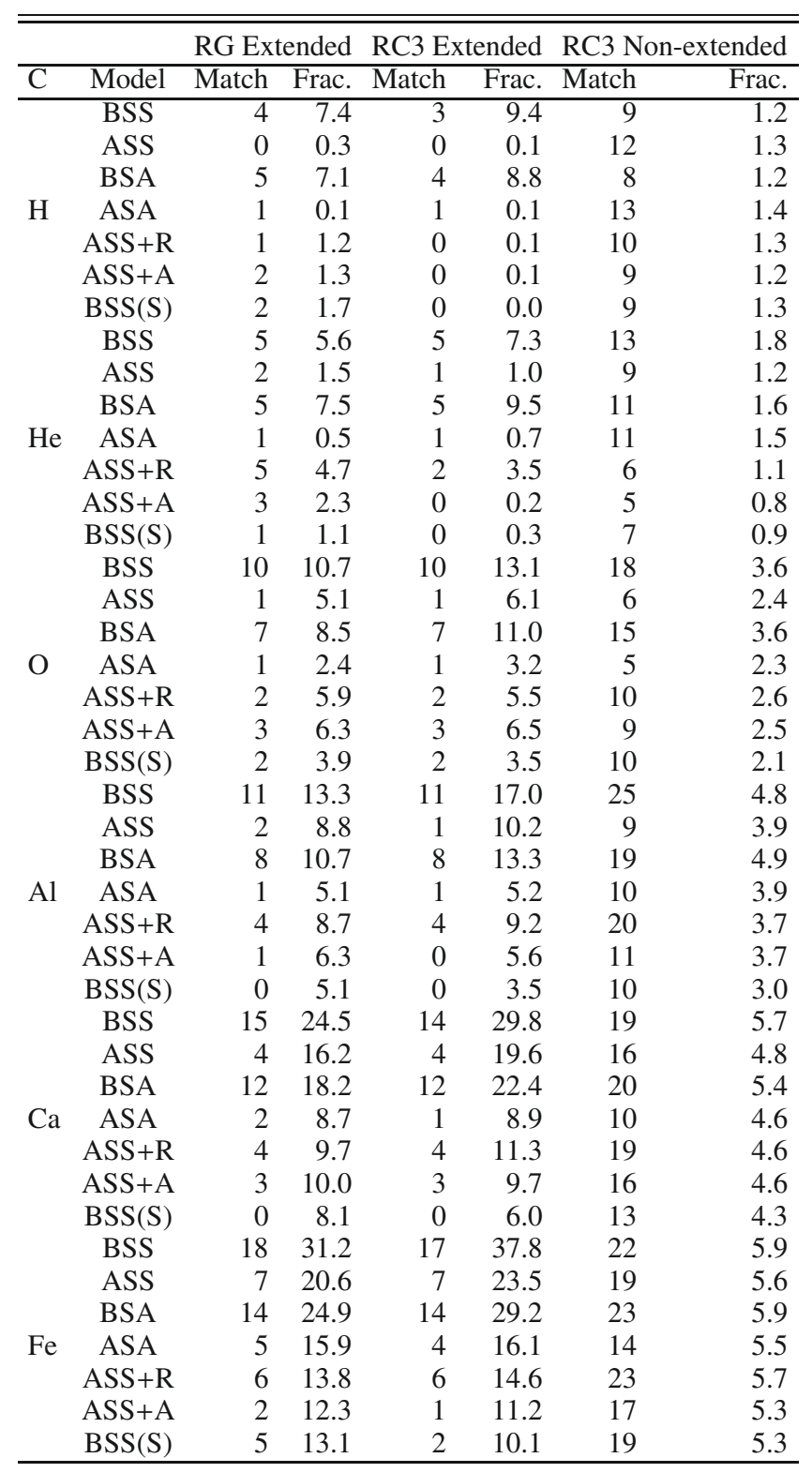

Notes. This table summarizes the match statistics between UHECRs detected by the Pierre Auger Observatory and three samples of ellipticals at $D \leq 75 \mathrm{Mpc}$ : all 15 nearby extended ( $L L S \geq 180 \mathrm{kpc}$ ) radiogalaxies (Cols. 3 and 4), all ellipticals from the RC3 catalog with extended $(L L S \geq 180 \mathrm{kpc}$ ) radio structure (Cols. 5 and 6) and a matched sample of ellipticals from the RC3 catalog without extended radio structure (Cols. 7 and 8; see text). Columns 1 and 2 list the UHECR composition and GMF model. The two columns for each sample of ellipticals list the number of UHECRs (of the total of 27) that can be matched (see text) to some member of the sample, and the fraction of the total (1350) Monte Carlo points that fall within the match distance-limit of the sample's galaxies.

of $2.5^{\circ}$. At heavy compositions, a large fraction of UHECRs are matched to nearby extended radiogalaxies.

For proton-UHECRs, the BS-S and BS-A models among the seven models tested maintain a strong correlation between UHECRs and radiogalaxies in the Cen A region (Figs. 1 to 5). After accounting for deflections within the BS-S and BS-A models, we find a stronger concentration of UHECRs around Cen A (the UHECR detected toward Cen B is now also deflected up to Cen A) and the radiogalaxy WKK 4432. Applying deflections in the models of S08 does not result in close matches between UHECRs and radiogalaxies in this region, though adding a dipole field to these models significantly improves the matches. In the case of the three matched events near NGC 7626, CGCG 413-019, and CGCG 514-050 (see Nagar \& Matulich 2008), all of BS-S, BS-A, and AS-S are acceptable for UHECRradiogalaxy matches; however, the S08 models - with or without a dipole component - do not provide consistently good matches.

For many other UHECRs, in Figs. 1 to 5 we do not see obvious matches between the Earth-arrival direction of UHECRs and nearby or intermediate (red and blue symbols) galaxies with radio jets. However, accounting for deflections by the GMF suggests several new potential matches between protonUHECRs and extended radio jets. Some interesting potential matches between galaxies with distance $<200 \mathrm{Mpc}$ and jet structures larger than $180 \mathrm{kpc}$ are worth noting: (a) we see possible matches between NGC 1275 and two AGASA-detected UHECRs; (b) CGCG 403-019, apart from a direct match to a PAO-detected UHECR, is potentially the source of three additional AGASA-detected UHECRs; (c) the extragalactic gammaray source with the highest maximum flux at HESS, Mrk 421, matches an AGASA UHECR; and (d) the BL Lacs H 2356-309 and 1ES 0347-121, both detected by HESS, can also be matched to AGASA events. We note that some of the AGASA events mentioned above do not appear in the figures because their $30 \%$ reduced energies are below $56 \mathrm{EeV}$.

To posit that the majority of UHECRs originate in galaxies with extended radio jets would require at least one of the following: (a) a stronger, or more ordered, intra-cluster and intergalactic magnetic field that produces extragalactic deflections greater than the current estimate (e.g., Harari et al. 2002; Dolag et al. 2004) of a few degrees for UHECRs; (b) a larger mean free path or lower energy loss than current estimates of the GZK effect, such that more distant radiogalaxies can contribute to the detected UHECR events; and/or (c) a large fraction of heavy composition UHECRs. The higher deflections of heavy UHECRs in Galactic and extragalactic magnetic fields produce differences of several tens of degrees between the out-of-Galaxy and Earth-arrival directions. Results on UHECR composition from the Auger collaboration infer that this scenario is likely (PA09a; PA09b).

Given the small sample size of known UHECRs, it is difficult to evaluate how the inclusion of UHECR deflections in the GMF affects the correlation between UHECRs and nearby massive spiral galaxies (Ghisellini et al. 2008) and is best left to later analysis with a larger number of UHECRs. Our comparisons of UHECR outside-the-Galaxy arrival directions with both the DRAGN sample and the several catalogs of gamma-ray sources also uncovered individually interesting coincidences. However, their statistical significance is difficult to evaluate and we do not discuss them here.

\subsection{Source populations: nearby extended radiogalaxies versus nearby elliptical galaxies}

Given the match between a subset of UHECRs and nearby extended radiogalaxies, it is relevant to test whether the potential association comes directly from the extended radio emission or some underlying material associated with massive elliptical galaxies, for e.g., luminous or dark matter concentrations. As a preliminary test, we used the RC3 catalog (Corwin et al. 1994) to extract all nearby $(D \leq 75 \mathrm{Mpc})$ elliptical galaxies. From this subset, we identified all nearby $(D \leq 75 \mathrm{Mpc})$ RC3 ellipticals 
with extended radio jets and lobes, obtaining a list of 11 galaxies that includes all of our 15 nearby extended radiogalaxies except NGC1275, Cen B, WKK 4552, and CGCG 514-050. The RC3 parameters of this subset of ellipticals satisfy the following limits: distance $D \leq 75 \mathrm{Mpc}$, morphological type $-5 \leq T \leq-2$, apparent $B$-band magnitude $7.69 \leq m_{B} \leq 13.38$, absolute $B$-band magnitude $-22.6 \leq M_{B} \leq-19.9$, and size of $1.2 \leq D_{25} \leq 2.42$, $0.02 \leq R_{25} \leq 0.2$, and $1.21 \leq D_{0} \leq 2.45$. We then selected a control sample of all RC3 ellipticals without extended radio jets and lobes that satisfied the above limits to RC3 measured parameters. This resulted in a control sample of 183 "radio-compact" ellipticals. Histograms comparing the distribution of distance, absolute magnitude, size, and angular separation from Cen A of the two samples are shown in Fig. 6. The two samples are reasonably well matched with a small tendency for the radio-extended ellipticals to have slightly higher absolute magnitudes and to be on average slightly closer to Cen A

A comparison of the statistics of matches of the two elliptical samples to the outside-the-Galaxy arrival directions of PAO UHECRs is shown in Table 5. When normalized for the number of galaxies in each sample, the matches between UHECRs and extended radiogalaxies is significantly higher than in the case of radio-compact ellipticals. This difference is most extreme in the case of the BS-S and BS-A models.

\subsection{Source populations: potential Galactic plane sources}

Very few UHECRs have been detected by PAO and AGASA in the region within $10^{\circ}$ of the Galactic plane. After including deflections in the GMF, however, several "Galactic-plane-crossing UHECRs" are found in most GMF models. In particular, the BS$\mathrm{S}$ and AS-S models, and the models of S08 with or without a dipole component, tend to move both the trajectories and out-ofGalaxy arrival directions of several UHECRs at $-30^{\circ}<b<0^{\circ}$ towards the Galactic plane.

We searched for potential matches between the UHECR trajectories within the Galaxy and objects from the catalogs of Galactic microquasars and Galactic magnetars. For the GMF models used, two or three UHECRs have trajectories that pass close (within $1 \mathrm{kpc}$ ) to a SGR or AXP: in the case of SGR 1900+14, a close match is obtained for all models except the S08 models without a dipole component. In the case of SGR 1627-41, deflections for the models AS-S and AS-A lead to a connection with the mysterious close pair of events detected by PAO. Several UHECR trajectories also pass close to confirmed or candidate Galactic microquasars and HESS-detected Galactic sources (e.g., Vela X and Vela Junior). Of course, fine tuning of the normalizations of the model permit closer matches, given the many free parameters (not least the uncertain distances to these Galactic sources and the mass of the UHECR). We thus do not attempt a detailed study of the coincidences, but merely note that some UHECRs potentially cross the Galactic plane near candidate UHECR sources.

\section{Discussion and concluding remarks}

The UHECR deflection simulations presented here and the derived outside-the-Galaxy arrival directions of UHECRs highlight several interesting points. For light (proton or helium) UHECRs, the relatively small dispersions in the outside-the-Galaxy arrival directions for a specific UHECR and GMF model confirm that tantalizing insights into the source population of UHECRs can be gained using a small sample of UHECRs. While heavy composition UHECRs experience deflections of several tens of de- grees, the concentration of outside-the-Galaxy arrival directions in the quadrant containing the SuperGalactic plane near Cen A also allows source population constraints. In contrast, selecting one or a few UHECR source populations permits an evaluation of competing models of the ordered GMF.

For light compositions, the trajectories of several UHECRs pass close to or through the Galactic plane within several GMF models. Interestingly, several pass relatively close to SGRs, AXPs, and/or microquasars in the Galaxy. Ghisellini et al. (2008) argued that magnetars in nearby galaxies could be responsible for the PAO-detected UHECRs. The integrated flux of $\mathrm{HI}$ emission from our Galaxy is greater than the summed integrated HI fluxes of all massive spirals in the HIPASS survey considered by Ghisellini et al. (2008). If UHECRs originate in magnetars in their steady state - rather than in formation then it would not be surprising to detect some magnetar-related UHECR events in the Galaxy.

In Nagar \& Matulich (2008), we claimed that a correlation exists between nearby galaxies with extended radio jets and the Earth-arrival direction of a subset of UHECRs. By fortuitous chance, these matched pairs lie along the lines of Galactic longitude that experience the least deflection by axisymmetric ordered Galactic magnetic fields. The correlation is strengthened by the results presented here. Accounting for deflections by BS-S and BS-A GMFs does not significantly change the previously claimed matches in the case of proton UHECRs. Using the BS-S model instead concentrates proton UHECRs closer to the nucleus of Cen A, and also ensures several new UHECRradiogalaxy matches. The models of S08, with an added dipole GMF component, also maintain the previous matched pairs. Both the nearest radiogalaxy and the nearest radio-extended BL Lac are potentially sources of multiple UHECRs. It is possible but unlikely that all UHECRs originate in Cen A. In the optimal case, i.e., when choosing the most convenient global GMF model for any given match, about $30 \%$ of UHECRs outside the range $-25^{\circ}<b<0^{\circ}$ could be matched to a galaxy with extended radio jets or an extragalactic gamma-ray source in the case of proton only UHECRs. For heavy composition or variable composition UHECRs, we cannot rule out that all UHECRs originate in nearby galaxies with extended radio jets. We have in particular noted several interesting and new matches between UHECRs and radiogalaxies for UHECR compositions near oxygen.

A remaining question is whether the correlation between radiogalaxies and UHECRs depends directly on the presence of extended radio jets and lobes or whether both trace an underlying source population. We have briefly addressed this issue in a comparative test of nearby ellipticals with and without extended radio structure. We find that there is a strong indication that, within the BS-S and BS-A models of the GMF, the correlation with nearby extended radiogalaxies is directly related to the radio jets rather than to an underlying source population traced by massive elliptical galaxies.

If radiogalaxies, or sources traced by radiogalaxies, are responsible for a subset of UHECRs, a BS-S, or BS-A model for the GMF is supported towards the Cen A region, i.e., the northern hemisphere of the fourth quadrant. The BS-S (but not the BS-A) model also allows a match between some UHECRs and Galactic plane sources. The models of S08 are consistent with both of the above only after we have added a dipole field component (see Sect. 2 again for why this dipole addition is not necessarily a self-consistent step).

Finally, we remark that this work identifies the subset of UHECRs that can be matched to extended radiogalaxies using a light composition $(\mathrm{H}$ or $\mathrm{He}$ ) for UHECRs, and those that re- 
quire a medium to heavy composition (e.g., Table 1). The relative ratio of proton to heavy composition UHECRs then roughly agree with the composition mix suggested by PA09a and PA09b. It would be interesting to verify whether the $X_{\max }$ distributions of these two UHECR subsamples confirm this composition difference.

Acknowledgements. We acknowledge funding from ALMA 3016013, ALMA 3107015, ALMA 3108022, Fondecyt 1080324, BASAL PFB-06/2007, and the FONDAP Center for Astrophysics. This research has made use of the NASA/IPAC Extragalactic Database (NED) which is operated by the Jet Propulsion Laboratory, California Institute of Technology, under contract with the National Aeronautics and Space Administration.

\section{References}

Abraham, J., Aglietta, M., Aguirre, I. C., J., et al. 2004, for the Pierre Auger Collaboration, Nucl. Instr. and Meth. A523, 50

The Pierre Auger Collaboration: Abraham, J., Abreu, P., Aglietta, M., et al. 2007, for the Pierre Auger Collaboration, Science, 318, 938 (PA07)

The Pierre Auger Collaboration: Abraham, J., Abreu, P., Aglietta, M., et al. 2008, Astropart. Phys., 29, 188 (PA08)

The Pierre Auger Collaboration: Abraham, J., Abreu, P., Aglietta, M., et al. 2009a, contribution to 31st ICRC, July 2009 [arXiv: 0906.2319] (PA09a)

The Pierre Auger Collaboration: Abraham, J., Abreu, P., Aglietta, M., et al. 2009b, contribution to 31st ICRC, July 2009 [arXiv: 0906.2347] (PA09b)

Beck, R. 2001, Space Sci. Rev., 99, 243

Beck, R. 2008, Amer. Instit. Phys. Conf. Ser., 1085, 83

Beck, R. 2009, Ap\&SS, 320, 77

Bock, D., Large, M. I., \& Sadler, E. M. 1999, AJ, 117, 1578

Bodaghee, A., et al. 2007, VizieR Online Data Catalog, 346, 70585

Brown, J. C., Haverkorn, M., Gaensler, B. M., et al. 2007, ApJ, 663, 258

Combi, J. A., Albacete-Colombo, J. F., \& Martí, J. 2008, A\&A, 477, 125

Condon, J. J., Cotton, W. D., Greisen, E. W., et al. 1998, AJ, 115, 1693

Corwin, H. G., Jr., Buta, R. J., \& de Vaucouleurs, G. 1994, AJ, 108, 2128

Cuesta, A. J., \& Prada, F. 2009, MNRAS, submitted [arXiv: 0910.2702]

de Pasquale, M., et al. 2006, VizieR Online Data Catalog, 345, 50813

Dolag, K., Grasso, D., Springel, V., et al. 2004, Nuclear Physics B Proceedings Supplements, 136, 234

George, M. R., Fabian, A. C., Baumgartner, W. H., Mushotzky, R. F., \& Tueller, J. 2008, MNRAS, 388, L59

Ghisellini, G., Ghirlanda, G., Tavecchio, F., Fraternali, F., \& Pareschi, G. 2008, MNRAS, 390, L88

Greisen, K. 1966, Phy. Rev. Lett, 16, 748

Han, J. 2002, Astrophysical Polarized Backgrounds, 609, 96

Han, J. L. 2008, Nuclear Physics B Proceedings Supplements, 175, 62
Han, J. L. 2007, IAU Symp., 242, 55

Han, J. 2009, IAU Symp., 259, 455

Harari, D., Mollerach, S., \& Roulet, E. 2002, Journal of High Energy Physics, 7, 6

Hartman, R. C., Bertsch, D. L., Bloom, S. D. et al. 1999, ApJS, 123, 79

Hayashida, N., Honda, N., Inoue, K., et al. 2000, unpublished [arXiv: astro-ph/0008102]

Heiles, C. 1996, ApJ, 462, 316

Hillas, A. M. 1999, Nuclear Physics B Proceedings Supplements, 75, 109

Hofmann, W. 2005, International Cosmic Ray Conference, 10, 97

Hooper, D., \& Taylor, A. M. 2010, Astropart. Phys., 33, 151 [arXiv: 0910.1842]

Kachelreiss, M. 2008, lecture notes on high energy cosmic rays [arXiv:astro-ph/0801.4376]

Kachelrieß, M., Serpico, P. D., \& Teshima, M. 2007, Astropart. Phys., 26, 378

Liu, F. K., \& Zhang, Y. H. 2002, A\&A, 381, 757

Laing, R. A., Riley, J. M., \& Longair, M. S. 1983, MNRAS, 204, 151

Matthews, J. N. 2007, Collicers to Cosmic Rays, 928, 47

Men, H., Ferrière, K., \& Han, J. L. 2008, A\&A, 486, 819

Meyer, M. J., Zwaan, M. A., Webster, R. L., et al. 2004, MNRAS, 350, 1195

Nagar, N. M., \& Matulich, J. 2008, A\&A, 488, 879

Noutsos, A., Johnston, S., Kramer, M., et al. 2008, MNRAS, 386, 1881

Paredes, J. M. 2005, Chin. J. Astron. Astrophys. Suppl., 5, 121

Prouza, M., \& Šmída, R. 2003, A\&A, 410, 1

Rachen, J. P., \& Biermann, P. L. 1993, A\&A, 272, 161

Romero, G. E., Combi, J. A., Perez Bergliaffa, S. E., et al. 1996, Astropart. Phys., 5,279

Ryu, D., Kang, H., \& Das, S. 2009, contribution to 31st ICRC, July 2009 [arXiv:0906.4850]

Sowards-Emmerd, D., Romani, R. W., \& Michelson, P. F. 2003, ApJ, 590, 109

Sowards-Emmerd, D., Romani, R. W., Michelson, P. F., Healey, S. E., \& Nolan, P. L. 2005, ApJ, 626, 95

Sowards-Emmerd, D., Romani, R. W., Michelson, P. F., et al. 2004, ApJ, 609, 564

Stanev, T. 1997, ApJ, 479, 290

Stanev, T. 2004, C. R. Physique 5, 453

Sun, X. H., Reich, W., Waelkens, A., et al. 2008, A\&A, 477, 573 (S08)

Takami, H., \& Sato, K. 2008, ApJ, 681, 1279

Taylor, J. H., \& Cordes, J. M. 1993, ApJ, 411, 674

Tinyakov, P. G., \& Tkachev, I. I. 2002, Astrop. Phys., 18, 165

Takeda, M., Hayashida, N., Honda, K., et al. 1999, ApJ, 522, 225

Vetere, L., Soffitta, P., Massaro, E., Giommi, P., \& Costa, E. 2007, VizieR Online Data Catalog, 347, 30347

Wainscoat, R. J., Cohen, M., Volk, K., Walker, H. J., \& Schwartz, D. E. 1992, ApJS, 83, 111

Wong, O. I., Ryan-Weber, E. V., Garcia-Appadoo, D. A., et al. 2006, MNRAS, 371,1855

Woods, P. M., \& Thompson, C. 2006, Compact stellar X-ray sources, 547

Zatsepin, G. T., \& Kuzmin, V. A., Soviet Phys. JETP, 4, 78 
N. M. Nagar and J. Matulich: UHECRs: galactic magnetic field deflections

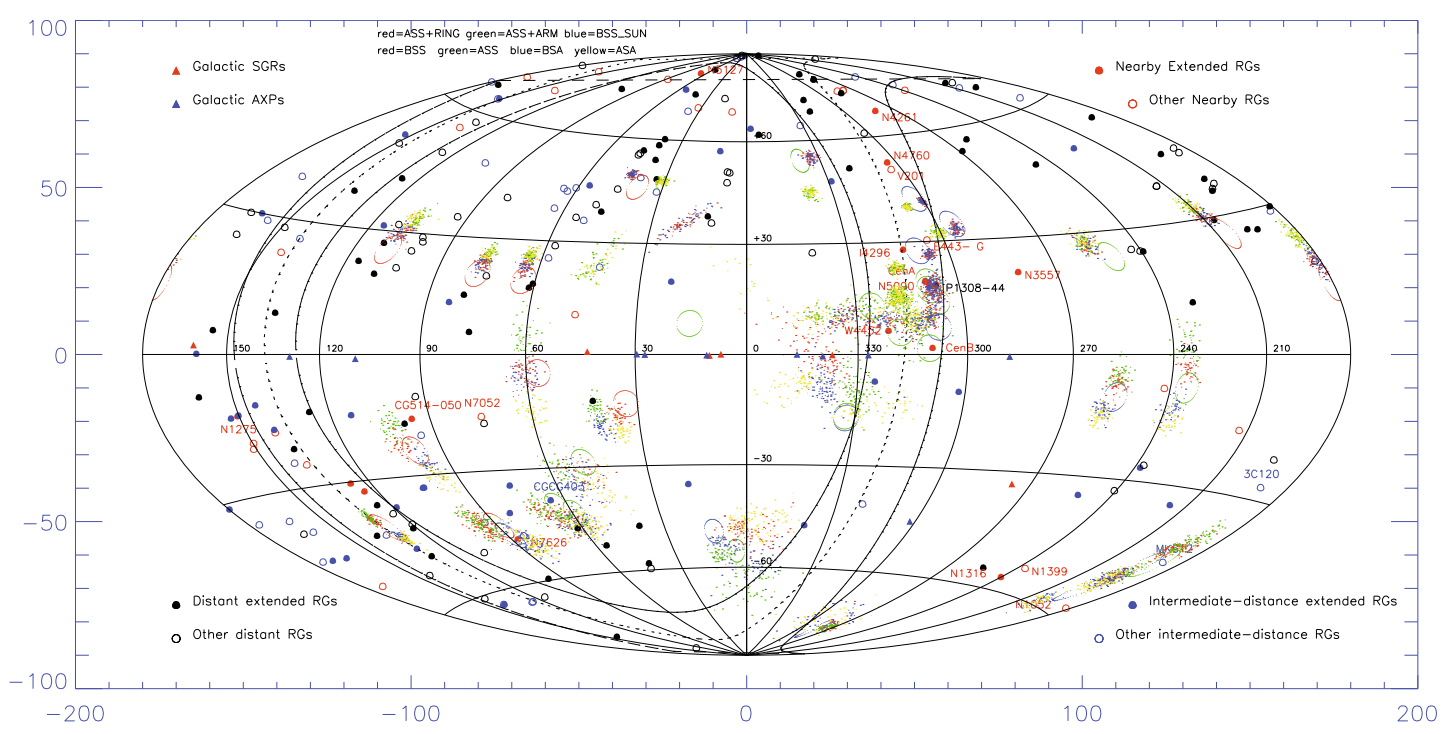

Fig. 2. Same as Fig. 1 for the three new models proposed by S08: AS-S+RING (small red dots), AS-S+ARM (small green dots), and BS-S (small blue dots). Here, as in S08, no ordered dipole field component was used.

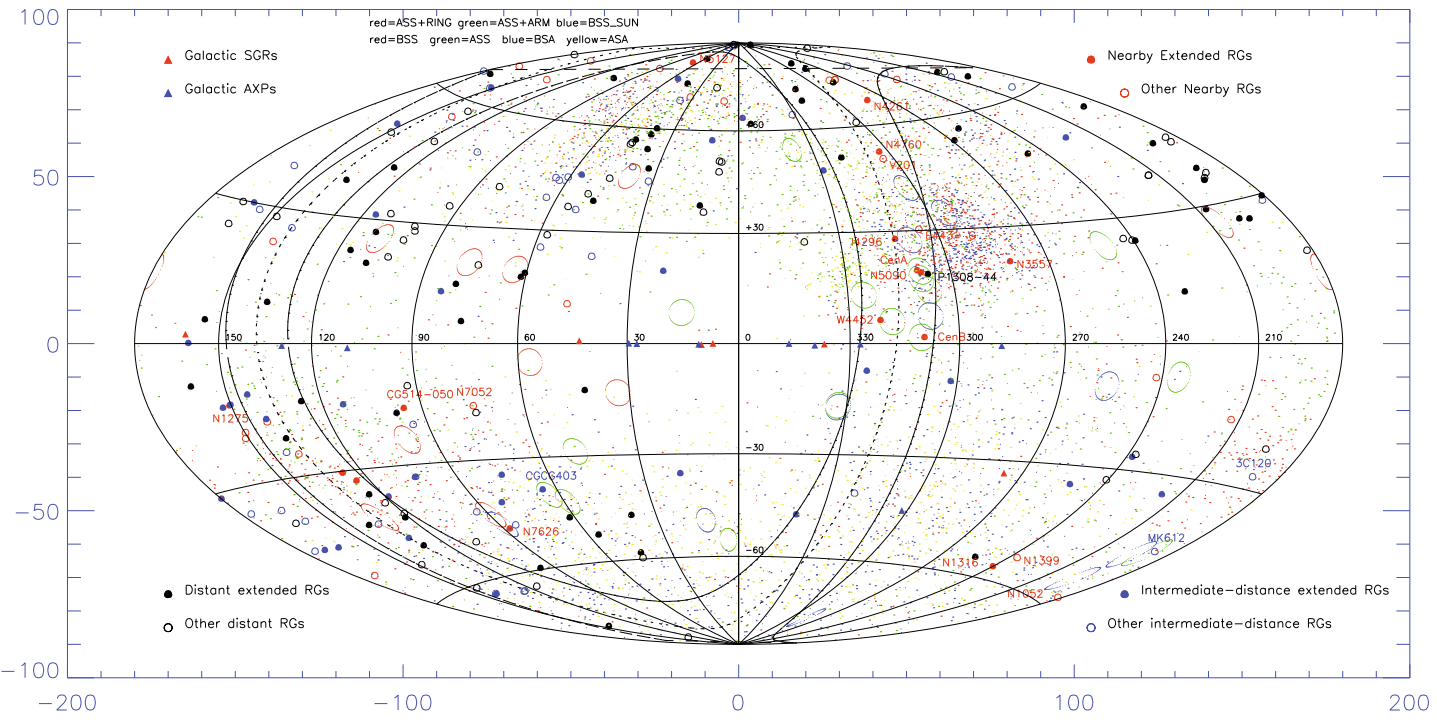

Fig. 4. Same as Fig. 1 but for a UHECR composition of oxygen.

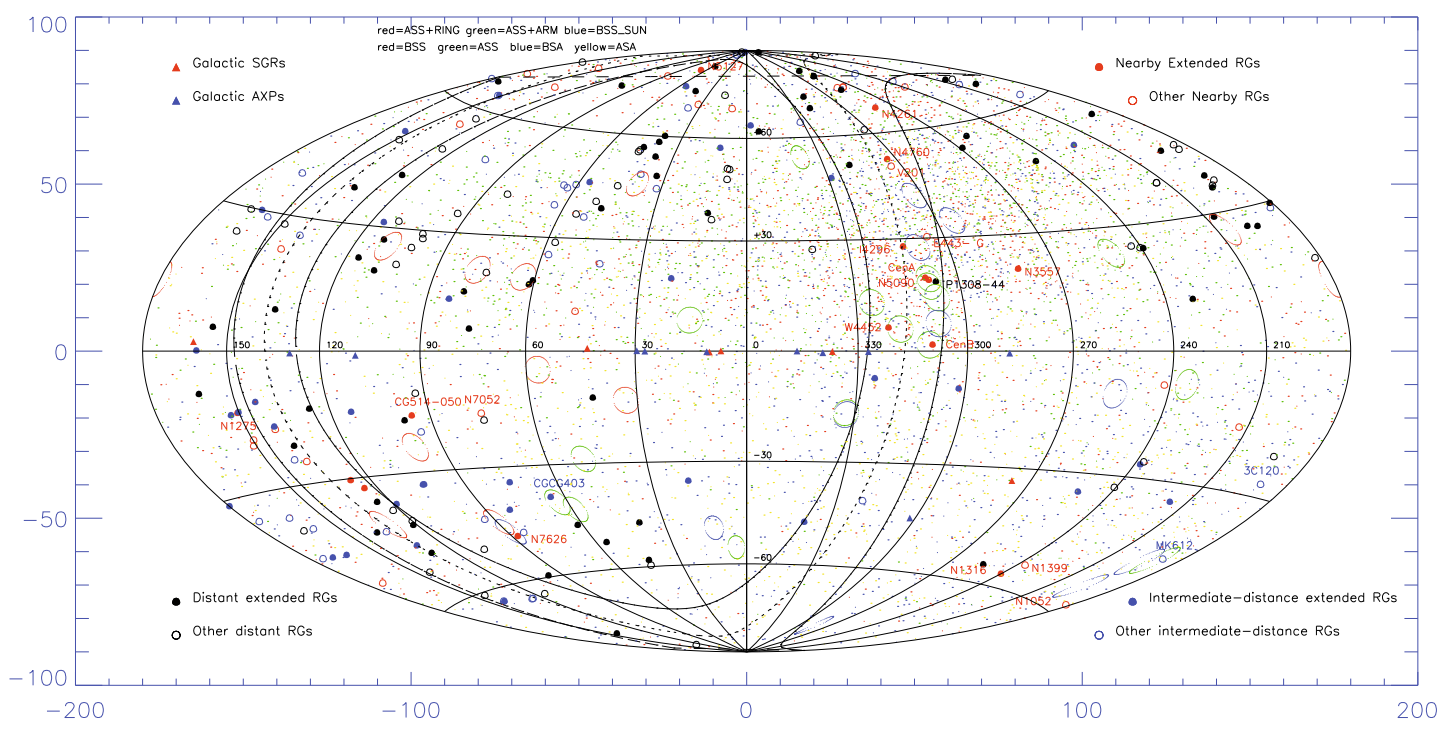

Fig. 5. Same as Fig. 1 but for a UHECR composition of iron. 